\title{
On the Application of Cooperative Communications in Renewable Energy Sources for Maximizing the Reliability of Power Distribution Networks
}

\author{
Theodoros Tsiftsis ${ }^{1}$, Nick Papanikolaou ${ }^{2}$, Michael Loupis $^{1}$ \\ and Vasilios Zarikas ${ }^{1}$ \\ ${ }^{1}$ Department of Electrical Engineering, Technological Educational Institute (T.E.I.) \\ of Lamia, 35100 Lamia, Greece; email:\{tsiftsis, mloupis, vzarikas@teilam.gr\} \\ ${ }^{2}$ Department of Electrical \& Computer Engineering, Democritus University of \\ Thrace, 67100 Kimmeria - Xanthi, Greece; email:\{npapanik@ee.duth.gr\}
}

Received: July 9, 2013; Accepted: September 25, 2013

\begin{abstract}
In this paper, the efficiency of cooperative communications in power distribution networks is investigated, where a number of renewable energy sources (RESs) are deployed. Specifically, any medium voltage (MV) bus of the power distribution network is considered as a wireless relay node where the information gathered from each MV bus can be reliably transmitted to a control station for further processing. Therefore, the power distribution network can be equivalently considered as a cooperative communication network. The average bit error rate of the proposed cooperative diversity network is studied and a closedform lower bound is analytically derived. Numerical results corroborate the tightness of the proposed bound compared to the Monte Carlo simulations. The proposed wireless communication scheme enables the increase of the RES penetration level in distribution networks, improving so RES exploitation.
\end{abstract}

Keywords: Average error probability, cooperative diversity, multihop network, power distribution network, renewable energy sources.

Journal of Green Engineering Vol. 3, 403-420.

doi: doi: 10.13052/jge1904-4720.343

(c) 2013 River Publishers. All rights reserved. 


\section{Introduction}

\subsection{Renewable Energy Sources}

The last decades witnessed rapid worldwide developments in the domain of electric power generation. Societies have become particularly concerned about environmental issues with ecological movements demonstrating significant activity on the need for immediate solutions. As a result, political organizations established extensive frameworks of support towards this promising sector $[1,2,3,4]$. Based on this, various RESs have been largely incorporated into power systems. More specifically, besides traditional employing of hydroelectric plants, the most significant RES contributor is solar energy which is exploited mainly by means of large wind farms (WF) with power levels between tens and hundreds of mega-watts (MW) [1,2]. To this effect, a sufficient increase of penetration level of RES in power systems is rather necessary either as a complementary energy source in the energy market or as main unit for microgrids. This task is among the top priorities of power generation policies and has attracted the interest of both academic and industrial sector, however numerous issues need to be adequately addressed. These issues are related to the power generation and distribution where the close collaboration of several technical domains such, power electronics, automation, control and power network analysis is required [5, 6].

In more details, the centralized RES units typically infuse energy in the power networks of high and extra high voltage levels. Hence, the produced energy is subject to significant transmission and distribution losses as well as losses related to increased investment costs due to the required infrastructure in rural areas [7, 8]. As a result, various efficient and reliable power electronic conversion solutions and effective driving techniques have been developed for the connection of RES units to the distribution level $[9,10,11,12,13$, $14,15]$. However, these converters appear to have an important operating problem which burdens the adequate penetration of RES. Notably, technical specifications are not capable to account for the case of power distribution networks with high proportion of penetration levels of RES i.e. over $20 \%$ of the total installed electric power [16, 17].

Evidently, the capability of merging various production units is closely related to the corresponding coverage of the required power load. This is important given that RESs are not available for long time intervals both in annual and daily basis. For example, wind farms are not sufficiently productive during summer time or during periods with weak winds. To this end, satisfactory load coverage requires the installment of additional alternative power 
generation components and potentially adequate power storing units such as batteries, fuel cells and flying wheels. The latter is particularly necessary in cases where a structure constitutes an autonomous microgrid which connects to the power distribution network asynchronously $[18,19,20]$. Therefore, the design and structure of the corresponding inverters must meet the specifications for serving simultaneously the various power production sources. Furthermore, they are required to cooperate closely with the storing units and the distribution network by providing high quality power with high operation efficiency.

Moreover, the dynamic characteristics and the overall behavior of the aforementioned converter topologies must be quantified adequately in the context of transient operating conditions. This is particularly important since the performance of distribution networks is often affected by transient phenomena caused mainly by voltage disturbances due to short circuits and/or from coupling/decoupling of large loads as well as by resonance related issues caused by the presence of non-linear loads. Typical methods to combat network-related disturbances include the use of active filters (AFs), dynamic voltage restorers (DVRs) as well as traditional voltage support elements (transformers with on load tap chargers, multilevel capacitors) $[21,22,23,24,25,26,27]$. As a result, it is undoubtedly important to guarantee accurate prediction of the behavior of the power distribution network during transient conditions and its incorporation in system analyses for electric power transmission. This is particularly critical since such analyses constitute the basis for making decisions related to the stability of power systems and the corresponding procedures on their commercialization [28, 29]. Therefore, it is evident that the core distribution networks should be constantly aware of the characteristics of each connected RES unit so that power quality as well as the stability and efficiency of the network are ensured $[30,31,32,33,34]$.

\subsection{Cooperative Communications}

Cooperative communication is an emerging wireless communication technology that possesses distinct features in terms of deployment, connectivity and quality of service. Furthermore, cooperative networks have been shown to be a key contributor towards minimizing traditional requirements for fixed telecommunication infrastructure. To this effect, relaying techniques enable network connectivity where traditional architectures are impractical due to location constraints and can be applied to cellular, 
wireless local area networks (WLAN), and hybrid networks. The concept underlying cooperative communications is that the source-terminal communicates with the destination-terminal through a number of relaysterminals, so called nodes. This concept is also known as multihop relaying transmissions and based on this multihop networks are capable of broadening radio coverage without necessarily using large transmitting power [35], [36].

In general, cooperative networks are multihop communication networks where the destination-terminal combines the signals received from both source-terminal and relays [37, 38, 39, 40]. This is a particularly critical procedure as the signal combining aims to minimize the corresponding information errors in the presence of fading. It is recalled here that fading is the varying distortion of wireless propagated signals which are subject to reflection, diffraction, refraction and scattering phenomena and have been shown to affect the performance and stability of wireless communication systems. Therefore, it is evident that analyzing the performance of multihop wireless communication networks over fading channels has been an important field of research [35, 36, 37, 38, 39, 40] and the references therein. Indicatively, in [39] a tight performance bound for the probability of error in a cooperative network over Rayleigh fading channels has been proposed. Furthermore, in [41, 42], performance bounds for multihop wireless communication networks with blind (fixed gain) relays over Rice, Hoyt and Nakagami- $m$ fading channels have been derived.

In the same context, with the aid of the well-known inequality between harmonic and geometric means of positive random variables (RVs), an efficient performance bound for the end-to-end signal-to-noise ratio (SNR) of wireless multihop networks with cooperative diversity operating over independent and not necessarily identically distributed (i.n.i.d.) Nakagami$m$ fading channels was reported in [43]. Capitalizing on that the proposed bound is a product of arbitrary powers of statistically independent squared Nakagami-m (Gamma) RVs, a closed-form expression was derived for the corresponding moment generating function (MGF). Using this expression, closed-form lower bounds were deduced for the average error probability for different digital modulation formats. The offered results showed a significant improvement in BER of transmitted information which constitutes such multi-hop deployments highly efficient and robust. 


\subsection{Contribution}

The usefulness and challenges of RES were revisited in detail in Sec. I.A. Based on this, it became evident that is crucial for power distribution networks to possess constantly information on the state of the characteristics of each individual component of the involved RESs. This will ensure the stable and robust operation of networks which require a sufficient quality of the infused energy produced by RESs. This can be achieved in a reliably manner with the aid of cooperative communication. Specifically, by using the wireless topologies of relay systems, each RES can be represented by an equivalent wireless relay node which can receive and transmit information. As it will be shown, this concept increases the reliability of power distribution networks as decisions on coupling of certain RESs over other less suitable ones will be made upon exploiting accurate information about the necessary component parameters. In more details, the contributions of this work are the following:

- A cooperative communication topology for efficient communication between RESs units and the power distribution network is proposed.

- According to the requirements of the operational scenario, each RES unit acts as a wireless source relay terminal or relay node and the substation either as a source or destination node.

- Based on the proposed method, the power distribution network can be notified continuously about the status and characteristics of each RES unit, enabling it to make an effective decision on the setpoint of each RES unit.

- The employed cooperative communication topology takes into account the fading phenomenon and provides significant reduction of communication errors i.e. reduced bit-error-rate and outage probability.

- The proposed technique is potential to increase significantly the effective penetration of RESs in the power distribution networks and will ensure the required energy quality and security of the network.

- The power distribution network will be remarkably flexible as each RES unit will act as a dynamic adaptive component which will couple to the network when it meets the energy and stability requirements.

To the best of the Authors' knowledge, the proposed concept has not been reported in the open technical literature. 


\section{System Model}

A MV radial distribution network with high RES penetration level is shown in Fig. 1. Any MV bus of this network may have RES generation (in terms of active and reactive power), an energy storage unit (EB) and loads. Consequently, a wireless sensor has to be installed at each MV bus so as to collect and transmit all the necessary information to the Substation control room. This piece of information includes the following data:

- RES power generation (both active and reactive).

- RES unit availability (on a daily basis).

- EB unit state of charge.

- Load consumption (both active and reactive).

- MV bus rms voltage level and phase angle.

- RMS current level at the point of common coupling (PCC).

- Active power level at the PCC.

- Report on any voltage disturbance incident (overvoltage, undervoltage, voltage asymmetry, excessive harmonic distortion).

- Report on any fault incident as well as on the corresponding reaction of the protection scheme.

On the other hand, each MV bus may accept directives from the Substation control room through its wireless sensor, such us:

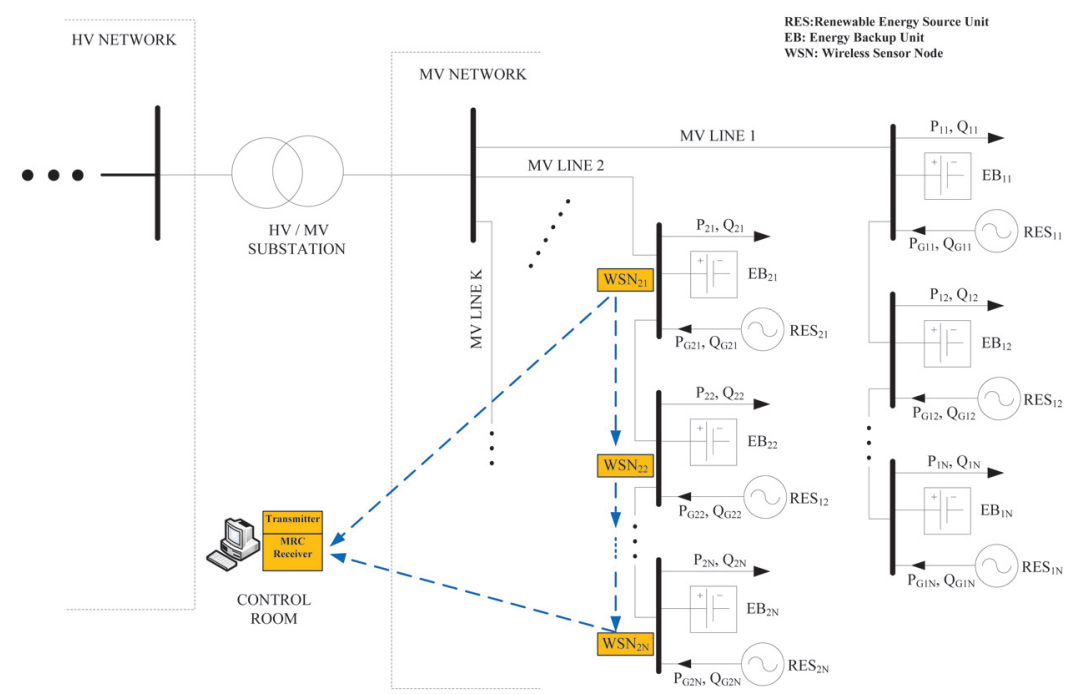

Figure 1 A MV radial distribution network with high RES penetration. 
- RES generation dispatching schemes.

- Load shedding schemes.

- Anti-islanding directions (connect/disconnect directions).

- Load forecast.

- RES generation forecast.

The MV radial distribution network with high RES installations can be equivalently be considered as a $N$-hop wireless communication network which operates over independent and not identically distributed (i.n.i.d.) Nakagami- $m$ fading channels. Specifically, it is assumed that the source terminal $\mathrm{S}\left(W S N_{21}\right)$ communicates with the destination terminal D (control room) through a direct link and via $N-1$ RES-nodes, $R_{1}\left(W S N_{22}\right), R_{2}\left(W S N_{23}\right), \ldots, R_{N-1}\left(W S N_{2 N}\right)$.

The control room is equipped with a transmit/receive antenna and can activate/deactivate each of WSN by transmitting a pilot signal. Therefore, if the information of a specific MV bus is needed, the control room activates the WSN assigned to this MV bus to transmit its information to the control room not only directly, but also via a number of predetermined WSNs. Each of the activated WSNs of the multihop communication network forwards the received information signal from its preceding node to the next one. The rest WSNs remain idle. Note that the control room is fully aware of the status of each WSN and can activate a certain number of WSNs to form an appropriate multihop communication network.

The WSNs can gather from RES nodes all the appropriate information as discussed above and play the role of intermediate non-regenerative relays from one hop to the next which are able to amplify-and-forward the information received by its preceding WSN. It is assumed that these WSNs can transmit over orthogonal time slots to ensure half-duplex operation and avoid any intersignal interference. Assuming that terminal $\mathrm{S}$ is transmitting a signal with an average power normalized to unity and maximal-ratio combining (MRC) at the destination terminal (control room), the end-to-end SNR, i.e., the SNR at the output of D, can be expressed as follows [44] :

$$
\gamma_{e n d}=\gamma_{0}+\tilde{\gamma}
$$

where

$$
\tilde{\gamma}=\prod_{i=1}^{N} v_{i}^{2} g_{i-1}^{2}\left(\sum_{i=1}^{N} \prod_{j=i+1}^{N} N_{0, i} g_{j-1}^{2} v_{j}^{2}\right)^{-1}
$$


where $\gamma_{0}$ is the instantaneous SNR between $\mathrm{S}$ and $\mathrm{D}, v_{i}$ is the fading amplitude of the $i$ th hop, $N_{0, i}$ is the one sided power spectral density at the input of the $i$ th relay, and $g_{i}$ is the gain of the $i$ th relay with $g_{0}=1$. Due to the fact that, $v_{i}$ follows the Nakagami- $m$ distribution, the corresponding instantaneous SNR, $\gamma_{i}$, defined as $\gamma_{i}=v_{i}^{2} / N_{0, i}$, is Gamma distributed with probability density function (PDF) expressed as [45]

$$
f_{\gamma_{i}}(\gamma)=\frac{m_{i}^{m_{i}} \gamma^{m_{i}-1} \exp \left(-\frac{m_{i} \gamma}{\bar{\gamma}_{i}}\right)}{\bar{\gamma}_{i}^{m_{i}} \Gamma\left(m_{i}\right)}
$$

where $m_{i} \geq 1 / 2$ is a parameter describing the fading severity of the $i$ th hop and $\bar{\gamma}_{i}$ is the average SNR, i.e., $\bar{\gamma}_{i}=E\left[v_{i}^{2}\right] / N_{0, i}$, with $E[\cdot]$ denoting expectation. By setting $\alpha_{i}=m_{i}$ and $\beta_{i}=\bar{\gamma}_{i} / m_{i}$ in (11), (3) is straightforwardly deduced. The corresponding gain can be expressed as [35]

$$
g_{i}^{2}=v_{i}^{-2}
$$

where the relay just amplifies the incoming signal with the inverse of the channel of the previous hop regardless of the fading state (i.e., the noise) of that hop. As mentioned in [35], such relays serve as benchmark for all practical multihop networks using non-regenerative relays. Furthermore, its performance in the high SNR region, is equal to the performance of the CSIassisted relays which satisfy the average power constraint, with an amplifying gain in [40, eq. (9)], namely,

$$
g_{i}^{2}=\left(v_{i}^{2}+N_{0, i}\right)^{-1} .
$$

By substituting (4) into (2), the end-to-end SNR becomes

$$
\gamma_{e n d}=\sum_{k=1}^{2} \tilde{\gamma_{k}}
$$

where

$$
\tilde{\gamma}_{k}= \begin{cases}\gamma_{0} & \text { for } \mathrm{k}=1 \\ \prod_{\iota=1}^{\mathrm{N}} \gamma_{i}\left(\sum_{i=1}^{N} \prod_{i \neq j=1}^{N} \gamma_{j}\right)^{-1} & \text { for } \mathrm{k}=2\end{cases}
$$

In order to study important performance metrics of the end-to-end SNR, (7) should be expressed in a more mathematically tractable form. This can be achieved with the aid of a tractable upper bound for (6) which is based on the 
well-known inequality between geometric $\mathcal{G}_{N}$ and harmonic $\mathcal{H}_{N}$ means of $N$ positive RVs $x_{1}, x_{2}, \ldots, x_{N}$, namely,

$$
\mathcal{H}_{N} \triangleq N\left(\sum_{i=1}^{N} 1 / x_{i}\right)^{-1} \leq \mathcal{G}_{N} \triangleq \prod_{i=1}^{N} x_{i}^{1 / N}
$$

By utilizing (7) and (8), an upper bound for the end-to-end SNR, $\gamma_{u b}$, for multihop networks with CSI-assisted relays is straightforwardly deduced that $\gamma_{\text {end }} \leq \gamma_{u b}$ with

$$
\gamma_{u b}=\gamma_{0}+\gamma_{a}=\gamma_{0}+\frac{1}{N} \prod_{i=1}^{N} \gamma_{i}^{1 / N} .
$$

Notably, the above results can be used effectively in the constant transmission of the state and characteristics of each RES. This can ensure the robust update of the power distribution network and can ensure efficient decision making with respect to coupling and de-coupling of RESs so that the required load demands are met.

\section{Average Error Probability}

Due to the statistical independence of $\gamma_{i}$ and $\gamma_{0}$, the moment-generatingfunction of the bound of the end-to-end SNR in (9) is expressed as,

$$
\mathcal{M}_{\gamma_{u b}}(s)=\mathcal{M}_{\gamma_{0}}(s) \mathcal{M}_{\gamma_{a}}(s)
$$

where $\mathcal{M}_{\gamma_{0}}(s)$ and $\mathcal{M}_{\gamma_{a}}(s)$ are the MGFs of $\gamma_{0}$ and $\frac{1}{N} \prod_{i=1}^{N} \gamma_{i}{ }^{1 / N}$, respectively. The MGF, $\mathcal{M}_{\gamma_{a}}$, is a product of arbitrary powers of $N$ Gamma RVs. To this effect, we let $\left\{X_{i}\right\}_{i=1}^{N}$ be $N$ independent, but not necessarily identically distributed (i.n.i.d.), Gamma RVs, with PDF given by

$$
f_{X_{i}}(x)=\frac{x^{\alpha_{i}-1}}{\beta_{i}^{\alpha_{i}} \Gamma\left(\alpha_{i}\right)} \exp \left(-\frac{x}{\beta_{i}}\right)
$$

where $\Gamma(\cdot)$ is the Gamma function [46, eq. (8.310.1)] and $\alpha_{i}, \beta_{i}$ be positive real numbers. Based on this, the MGF of the new RV $Y_{1}$ can be expressed as the product of arbitrary powers of $N$ RVs $X_{i}$, namely, $Y_{1} \triangleq \prod_{i=1}^{N} X_{i}^{\ell_{i} / k}$ with $\ell_{1}, \ell_{2}, \ldots, \ell_{N}$ and $k$, being positive integers. Importantly, the MGF of $Y_{1}$ can 


\section{T. Tsiftsis et al.}

be expressed in closed-form in terms of the Meijer G-special function [46, eq. (9.301)] as in [44]

$$
\mathcal{M}_{Y_{1}}(s)=\Lambda G_{r, k}^{k, r}\left[g(s) \mid \begin{array}{c}
Q_{\ell_{1}, 1-\alpha_{1}}, Q_{\ell_{2}, 1-\alpha_{2}}, \ldots, Q_{\ell_{N}, 1-\alpha_{N}} \\
Q_{k, 0}
\end{array}\right]
$$

where

$$
g(s)=\frac{(-1)^{k}(s / k)^{k}}{\prod_{i=1}^{N}\left(\beta_{i} \ell_{i}\right)^{-\ell_{i}}}
$$

and

$$
\Lambda=k^{1 / 2}(\sqrt{2 \pi})^{-r+N-k+1}\left(\prod_{i=1}^{N} \Gamma\left(\alpha_{i}\right)\right)^{-1} \prod_{i=1}^{N} \ell_{i}^{\alpha_{i}-1 / 2}
$$

where $r=\sum_{i=1}^{N} \ell_{i}, Q_{k, u} \triangleq u / k,(u+1) / k, \ldots,(u+k-1) / k$, with $u$ real. To this effect, by inserting (12) in (10) and the MGF expression of $\gamma_{0}$ presented in [45, Table 2.2], the MGF of $\gamma_{u b}$ can be expressed as

$$
\begin{array}{r}
\mathcal{M}_{\gamma_{u b}}(s)=\left(1-\frac{s \bar{\gamma}_{0}}{m}\right)^{-m} \frac{N^{1 / 2}}{(2 \pi)^{(N-1) / 2} \prod_{i=1}^{N} \Gamma\left(m_{i}\right)} \\
\times G_{N, N}^{N, N}\left[h(s) \mid \begin{array}{c}
m_{1}, m_{2}, \ldots, m_{N} \\
Q_{N, 0}
\end{array}\right]
\end{array}
$$

where

$$
h(s)=(-1)^{N}\left(s / N^{2}\right)^{N}\left(\prod_{i=1}^{N}\left(\bar{\gamma}_{i} / m_{i}\right)^{-1}\right)^{-1}
$$

It is noted here that with the aid of [47, eq. (18)], the Meijer's G-function can be written in terms of the more familiar generalized hypergeometric functions [46, eq. (9.14.1)]. Furthermore, it is recalled that both the Meijer's G-function and the hypergeometric functions are standard built-in functions in most commercial mathematical software packages such as Maple and Mathematica. As a result, their computational implementation is realized rather quickly and straightforwardly. 


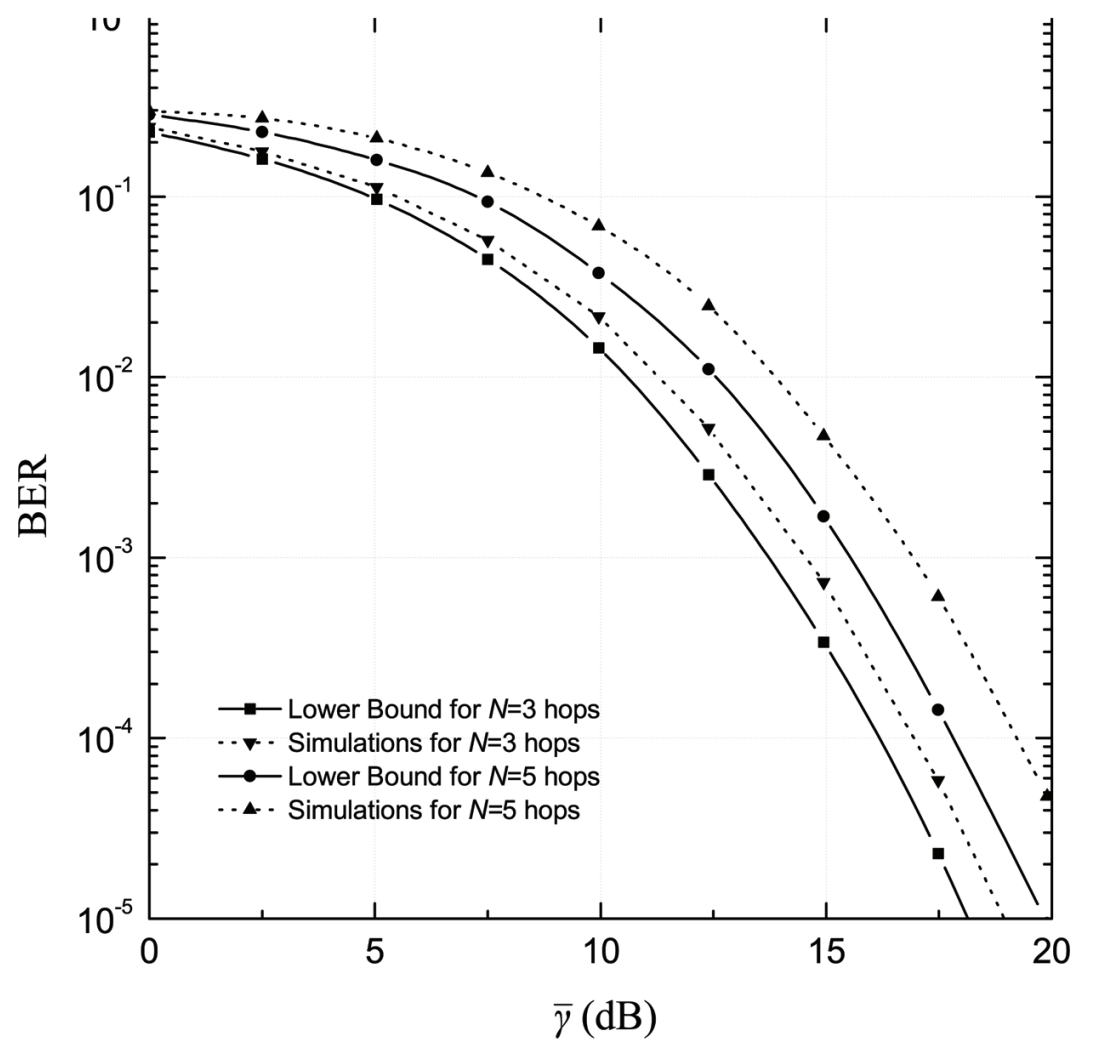

Figure 2 BER lower bounds for a cooperative multihop RES network $\left(\bar{\gamma}_{i}=\bar{\gamma}_{0}=\bar{\gamma}\right.$ and $m_{i}=m=2$ ).

\section{Numerical \& Simulation Results}

With the aid of the closed-form expression for the MGF of $\gamma_{u b}$ in (15), the average bit error probability (BER) can be evaluated according to the MGF-based approach for the performance evaluation of digital modulations over fading channels [45]. This can be achieved for a wide variety of digital modulation formats such as $M$-ary phase-shift keying ( $M$-PSK) and $M$-ary quadrature amplitude modulation ( $M$-QAM).

For the case of equal average SNR per hop (for all hops, $\bar{\gamma}_{i}=\bar{\gamma}_{0}=$ $\bar{\gamma})$, lower bounds for the BER for binary phase shift-keying (BPSK) of a cooperative multihop RES network are depicted for $N=3$ and $N=5$ hops. It is clearly shown that the average BER decreases substantially as the number of hops increases. Indicatively, for $\bar{\gamma}=12 \mathrm{~dB}$, the corresponding BER difference 


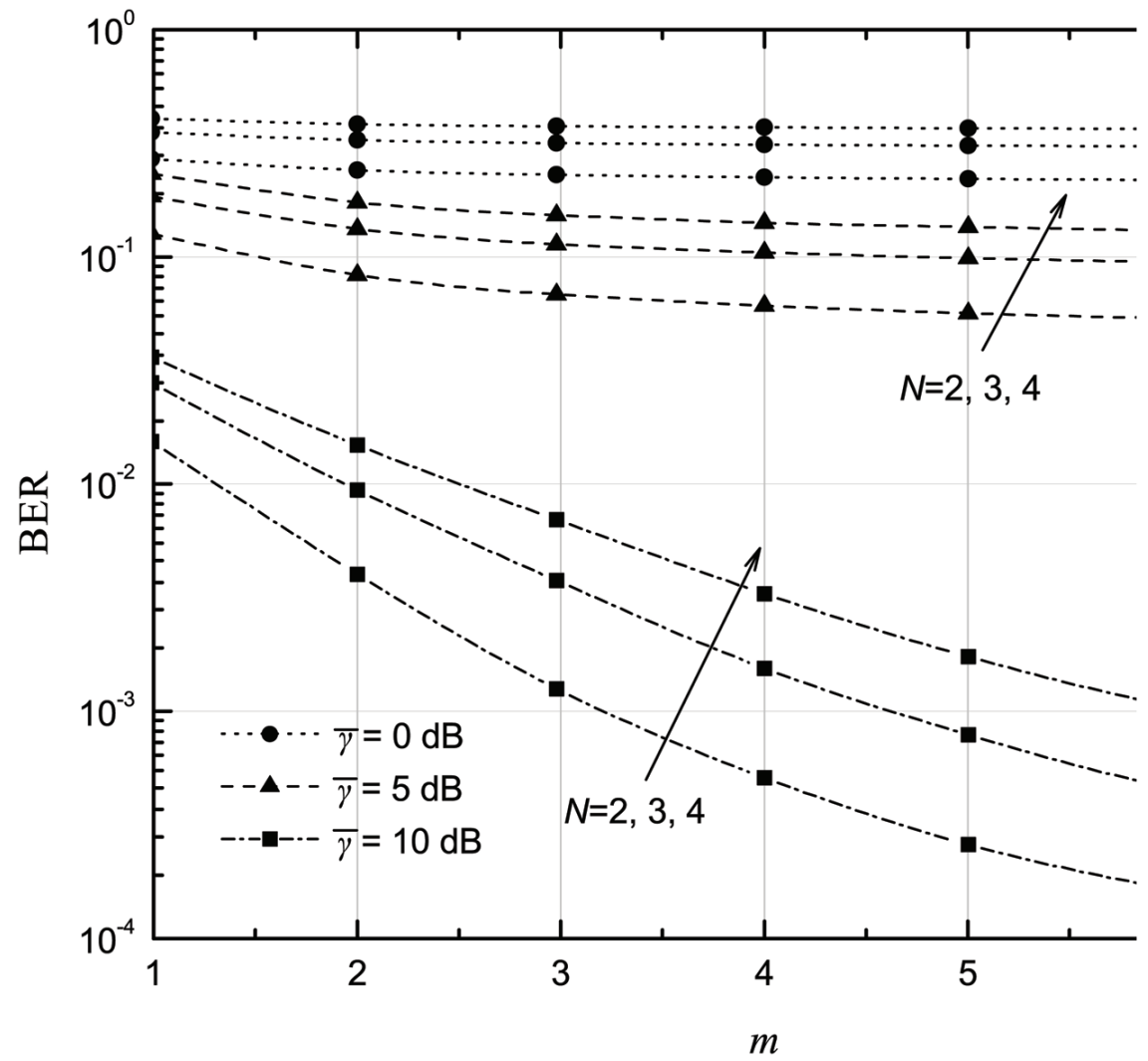

Figure 3 BER lower bounds for cooperative multihop RES network versus Nakagami fading parameter $m\left(\bar{\gamma}_{i}=\bar{\gamma}_{0}=\bar{\gamma}\right.$ and $\left.m_{i}=m\right)$.

is up to two orders of magnitude which demonstrates the effectiveness of this method. This is particularly important in power distribution networks that employ RESs as the required assessment on the characteristics and figures of each component can be transmitted remarkably accurately.

In the same context, Figure 3 illustrates the corresponding BER versus $m$ for the case of BPSK constellation. One can notice the effect of fading on the system performance. However, by comparing the two figures it immediately follows that the involved fading variations can be effectively combated by increasing the number of relay nodes. Since these nodes can be the RESs themselves, it is evident that deploying this concept in modern power generation systems and distribution networks can increase substantially their performance, stability and robustness. 


\section{Conclusions}

This work was devoted to the deployment possibilities of cooperative communication networks in power distribution networks with increased RES penetration level. Given the high requirements of RESs for joining the network, it is important that the characteristics of each component are fully available upon making a decision on the operating conditions of each RES unit at a given scenario. It was extensively shown that this can be achieved by employing cooperative communication concepts where each MV bus (e.g., RES) can act as a wireless relay node. To this effect, the status of each RES unit is fully available at the power distribution network with high reliability, since the corresponding error rate is significantly low.

\section{Acknowledgment}

This work was supported by the Research Program DGRES (MIS 380360) within the Research Activity ARCHIMEDES III, funded by the NSRF 20072013, Greece.

\section{References}

[1] J. F. B. Mitchel. The Greenhouse Effect and Climate Chnage. http: //www. bom.gov.au/info/GreenhouseEffectAndClimateChange.pdf

[2] C. Campbell C and J. Laherrere. The end of cheap oil. Science American, 278:78-83, 1998.

[3] P. J. Runci. Energy R\&D in the European Union. Pacific Northwest National Laboratory, U.S. Department of Energy, Contract DE-AC06-76RLO 1830, May 1999, No PNNL12218, Internet Report.

[4] K. Delkis. Greece Action Plan. Renewable Electricity and Liberalised Markets, Joule-III, Project, ICCS/NTUA, 1999.

[5] S. B. Kjaer. State of the art analysis for the âE SolcelleInverterât ${ }^{\mathrm{TM}}$ project. Aalborg University, 2002.

[6] S.W.H. De Haan, H. Oldenkamp and E. J. Wildenbeest. Test results of a 130W AC Module; a modular solar ac power station. Proceedings of the IEEE Photovoltaic Specialist Conference (PSC'94), 1994.

[7] R. H. Wills, E. Hall, S. J. Strong, J. H. Wohlgemuth, The AC Photovoltaic Module, Proceedings of the IEEE 5th Photovoltaic Specialists Conference (PSC'96), Washington DC (USA), 1996.

[8] K. Kurokawa, K. Kamisako and T. Shimizu T. Conceptual considerations on PV systems composed of AC modules. Solar Energy Materials and Solar Cells, 47(1-4):243-250, 1997. 
[9] Z. Chen, J. M. Guerrero, and F. Blaabjerg. A Review of the State of the Art of Power Electronics for Wind Turbines. IEEE Transactions on Power Electronics, 24(8):18591875, 2009.

[10] R. K. Beheraa and W. Gaob. A Novel Controller Design for Grid-side Converter of Doubly Fed Induction Generator for Wind Power Interface: An Experimental Investigation, Electric Power Components and Systems. Electric Power Components and Systems, 38(14):1531-1545, 2010.

[11] H. Alatrash, A. Mensah, E. Mark, G. Haddad and J. Enslin. Generator Emulation Controls for Photovoltaic Inverters. IEEE Transactions on Smart Grid, 3(2):996-1011, 2012.

[12] E. H. Camm and S. E. Williams. Solar power plant design and interconnection. Proceedings of the 2011 IEEE Power and Energy Society General Meeting, 2011.

[13] C. Photong, C. Klumpner, and P. Wheeler. A current source inverter with series connected AC capacitors for photovoltaic application with grid fault ride through capability. Proccedings of the 35th Annual Conference of IEEE Industrial Electronics, 2009.

[14] C. Schauder. Impact of FERC 661-A and IEEE 1547 on Photovoltaic Inverter Design. Proceedings of the 2011 IEEE Power and Energy Society General Meeting, 2011.

[15] J. C. Vasquez, R. A. Mastromauro, J. M. Guerrero, S. Member, and M. Liserre. Voltage Support Provided by a Droop-Controlled Multifunctional Inverter. IEEE Transactions on Industrial Electronics, 56(11):4510-4519, 2009.

[16] L. Gertmar, L. Liljestrand, and H. Lendenmann. Wind Energy Powers-That-Be Successor Generation in Globalization. IEEE Transactions on Energy Conversion, 22(1):13-28, 2007.

[17] CIGRE (International Council on Large Electric Systems). Connection Criteria at the Distribution Network for Distributed Generation. 2005.

[18] R. Majumder, S. Chakrabarti, G. Ledwich, and A. Ghosh. Advanced Battery Storage Control for an Autonomous Microgrid. Electric Power Components and Systems, 41(2):157-181, 2013.

[19] M. Khederzadeh, and H. Maleki. Frequency Control of Microgrids in Autonomous Mode by a Novel Control Scheme Based on Droop Characteristics. Electric Power Components and Systems, 41(1):16-30, 2013.

[20] M. I. Marei, and M. H. Soliman. A Coordinated Voltage and Frequency Control of Inverter Based Distributed Generation and Distributed Energy Storage System for Autonomous Microgrids. Electric Power Components and Systems, 41(4):383-400, 2013.

[21] N. Athanasiadis. Power Quality Solutions for Voltage Sags Using Dynamic Voltage Restorers. Electric Power Components and Systems, 31(2):159-170, 2003.

[22] W. El-Khattam, A. Elnady, and M. M. A. Salama. Dynamic Voltage Restorer Cost Reduction in the Distributed Generation Environment. Electric Power Components and Systems, 32(6):611-626, 2004

[23] C. Venkatesh, D. V. S. S. Siva Sarma, and M. Sydulu. Mitigation of Voltage Sag/Swell Using Peak Detector Based Pulse Width Modulation Switched Autotransformer. Electric Power Components and Systems, 39(11):1117-1133, 2011.

[24] B. Singh, P. Jayaprakash, and D. P. Kothari. Three-Leg Voltage Source Converter Integrated with T-connected Transformer as Three-phase Four-wire Distribution Static Compensator for Power Quality Improvement. Electric Power Components and Systems, 37(8):817-831, 2009. 
[25] A. Ghamri, M. T. Benchouia, and A. Golea. Sliding-mode Control Based Three-phase Shunt Active Power Filter: Simulation and Experimentation. Electric Power Components and Systems, 40(4):383-398, 2012.

[26] K. M. Tsang, W. L. Chan, and Xin Tang. Multi-level Shunt Active Power Filter Using Modular Cascade H-bridge and Delay Firing. Electric Power Components and Systems, 41(6):605-618, 2013.

[27] N. Gupta, S. P. Singh, and R. C. Bansal. A Digital Signal Processor Based Performance Evaluation of Three-phase Four-wire Shunt Active Filter for Harmonic Elimination, Reactive Power Compensation, and Balancing of Non-linear Loads under Non-ideal Mains Voltages. Electric Power Components and Systems, 40(10):1119-1148, 2012.

[28] X. Xiong, and J. Ouyang. Modeling and Transient Behavior Analysis of an Inverter-based Microgrid. Electric Power Components and Systems, 40(1):112-130, 2011.

[29] B. Johnson, A. Davoudi, P. Chapman, and P. Sauer. A Unified Dynamic Characterization Framework for Microgrid Systems. Electric Power Components and Systems, 40(1):93$111,2011$.

[30] P. Rooij, M. Real, U. Moschella, T. Sample and M. Kardolus. 'Advanced Reliability Improvement of AC-Module (ARIA). Netherlands Energy Research Foundation ECN (co-ordinator), Research founded in part by the European Commission in the framework of the Non Nuclear Energy Programme JOULE III, Contract No JO3-CT97-0122, 1 July 1997 to 31 May 2000, http://www.ecn.nl/docs/library/report/2001/c01093.pdf.

[31] M. Kusakawa, H. Nagayoshi, K. Kamisako and K. Kurokawa. Further Improvement of a transformerless voltage-boosting inverter for AC modules. Solar Energy Materials \& Solar Cells, 67(1-4):379-387, 2001.

[32] A. Ch. Kyritsis, E. C. Tatakis and N. Papanikolaou. Optimum Design of the CurrentSource Flyback Inverter for Decentralized Grid-Connected Photovoltaic Systems. IEEE Transactions on energy conversion, 23(1):281-293, 2008.

[33] S. B. Kjaer, J. K. Pedersen and F. Blaabjerg. A Review of Single-Phase Grid-Connected Inverters for Photovoltaic Modules. IEEE Transactions on industry applications, 41(5):1292-1306, 2005.

[34] B. Brooks and C. Whitaker. CEC Guideline for the use of the Performance Test Protocol for Evaluating Inverters Used in Grid-Connected Photovoltaic Systems. Sandia Inverter Performance Test Procedures, 2005.

[35] M. O. Hasna, M.-S. Alouini. Outage probability of multihop transmission over Nakagami fading channels. IEEE Communications Letters 7(5):216-218, 2003.

[36] J. Boyer J, D. D. Falconer and H. Yanikomeroglu. Multihop diversity in wireless relaying channels. IEEE Transactions on Communications, 52(10):1820-1830, 2004.

[37] A. Sendonaris, E. Erkrip and B. Aazhang. User cooperation diversity. Part I: System description. IEEE Transactions on Communications 51(11):1927-1938, 2003.

[38] A. Sendonaris, E. Erkrip and B. Aazhang. User cooperation diversity. Part II: Implementation aspects and performance analysis. IEEE Transactions on Communications 51(11):1939-1948, 2003.

[39] P. A. Anghel and M. Kaveh. Exact symbol error probability of a cooperative network in a Rayleigh-fading environment. IEEE Transactions on Wireless Communications, 3(5):1416-1421, 2004. 
[40] J. N. Laneman, D. N. C. Tse and G. W. Wornell. Cooperative diversity in wireless networks efficient protocols and outage behaviour. IEEE Transactions on Information Theory, 50(12):18-22, 2004.

[41] G. K. Karagiannidis. Performance Bounds of Multihop Wireless Communications with Blind Relays over Generalized Fading Channels. IEEE Transactions on Wireless Communications, 5(3):498-503, 2006.

[42] G. K. Karagiannidis. Moments-based approach to the performance analysis of equal-gain diversity in Nakagami- $m$ fading. IEEE Transactions on Communications 52(5):685-690, 2004.

[43] T. A. Tsiftsis. Performance of wireless multihop communications systems with cooperative diversity over fading channels. International Journal of Communications Systems, Wiley, 21(5):559ât“"-565 2008.

[44] G. K. Karagiannidis GK, T. A. Tsiftsis and R. K. Mallik. Bounds for multihop realayed communications in Nakagami- $m$ fading channels. IEEE Transactions on Communications, 50(1):3062-3080, 2006.

[45] M. K. Simon and M.-S. Alouini. Digital Communication over Fading Channels (2nd edn). Wiley, New-York 2005.

[46] I. S. Gradshteyn and I. M. Ryzhik. Table of Integrals, Series, and Products. (6th edn). Academic, New York 2000.

[47] V. S. Adamchik and O. I. Marichev. The algorithm for calculating integrals of hypergeometric type functions and its realization in REDUCE system. Proceedings of the International Conference on Symbolic and Algebraic Computation, 1990.

\section{Biographies}

Theodoros Tsiftsis was born in Lamia, Greece, in November 1970. He received the degree in Physics from the Aristotle University of Thessaloniki, Greece, in 1993, and the M.Sc. degree in Digital Systems Engineering from the Heriot-Watt University, Edinburgh, Scotland, U.K., in 1995. Also, he received the M.Sc. degree in Decision Sciences from the Athens University of Economics and Business, Greece, in 2000 and the Ph.D. degree in Electrical Engineering from the University of Patras, Greece, in 2006. He is currently an Assistant Professor in the Department of Electrical Engineering at Technological Educational Institute of Lamia, Greece. His major research interests include relay assisted and cooperative communications, wireless communications over fading channels, wireless communications theory and optical wireless communications. He has published and presented more than 70 technical papers in scientific journals and international conferences. Dr. Tsiftsis acts as reviewer for several international journals and he served as Editor of the IEEE Transactions on Vehicular Technology and IET Communications. $\mathrm{He}$ is currently a member of the Editorial Boards of IEEE Transactions on Communications and IEEE Communications Letters. 
Nick Papanikolaou received the Diploma and the $\mathrm{PhD}$ degrees in Electrical Engineering by University of Patras, in 1998 and 2002 respectively. He has more than ten yearât ${ }^{\mathrm{TM}_{\mathrm{S}}}$ research activity in the fields of Power Electronics, Power Quality and Renewable Energy Sources. He is currently an Assistant Professor in the Department of Electrical \& Computer Engineering, Democritus University of Thrace, Xanthi, Greece. He has important working experience in Greek Electrical Power Industry âE " where he used to work as System Design Engineer âŁ " and he has published many research papers in international scientific journals and conferences, being cited in more than 200 papers by other research teams. Dr. Papanikolaou is a Senior Member of IEEE and a Member of the Technical Chamber of Greece and the Greek Union of Mechanical \& Electrical Engineers. He is regular reviewer in various international scientific journals, as well as in national research programs funded by the Greek General Secretary for Research and Technology.

Michael Loupis was born in Athens on 11/9/1962. He earned a Dipl.Ing. in Electrical Engineering from the University of Thessaloniki, Greece in 1986, an M.Sc. in Microprocessor Engineering from the University of Bradford, UK in 1987, a Dr.Ing. in Information Technology from the National Technical University of Athens (NTUA) in 1999 and an M.Sc. in Quality Assurance from the Greek Open University in 2006. He is currently an Assistant Professor with the Lamia Institute of Technology, Department of Electrical Engineering, Greece. His current research interests include software and modelling tools for embedded systems and design tools for renewable energy systems and energy management. Prof. Loupis is a Senior Member of IEEE, a Member of the Technical Chamber of Greece, the Greek Union of Electrical and Mechanical Engineers and the Greek Association of Computer Engineers.

Vasilios Zarikas is a member of the Academic staff of the Department of Electrical Engineering at Lamia Institute of Technology (TEI Lamias). He received his Ph.D. in Theoretical Physics and Applied Mathematics from the University of Newcastle upon Tyne, England, U.K. and an M.Sc. degree from the University of Sussex, U.K. A First class Bachelor degree in Physics was received from the University of Thessaloniki, Greece. He was a postdoctoral researcher, a lecturer and an assistant researcher in several Universities (University of Athens-Dept. of Physics, Finance University of Athens, University of Crete - Mathematics Department and Foundation of Research and Technology FORTH-Heraclion Crete). He has received Ph.D. and M.Sc. Scholarships and assistantships as well as a research award. His research 
interests include Applied Mathematics, Applied Statistics on Technology and Medicine, Mathematical decision modeling, Bayesian networks, intelligent human computer interaction, mathematical physics, quantum field theory, cosmology and astroparticle physics. His research work in mathematical modeling consists of more than 35 fully refereed journal and conference papers (20 journals, one book) and it has been recognized by 170 citations. Furthermore, his research activity has been received the support of National and European research funding (active member of European research projects. More details can be found in http://www.ele.teilam.gr/zarikas.htm. 